\title{
Incorporating Negentropy in Saliency-based Search Free Car Number Plate Localization
}

\author{
Amin Safaei, Hongying L. Tang, and Saeid Sanei \\ Faculty of Engineering and Physical Sciences, University of Surrey \\ Guildford, United Kingdom \\ Email: a.safaei@surrey.ac.uk,h.tang@ surrey.ac.uk, s.sanei@surrey.ac.uk
}

\begin{abstract}
License plate localization algorithms aim to detect license plates within the scene. In this paper, a new algorithm is discussed where the necessary conditions are imposed into the saliency detection equations. Measures of distance between probability distributions such as negentropy finds the candidate license plates in the image and the Bayesian methodology exploits the a priori information to estimate the highest probability for each candidate. The proposed algorithm has been tested for three datasets, consisting of gray-scale and color images. A detection accuracy of $96 \%$ and an average execution time of $80 \mathrm{~ms}$ for the first dataset are the marked outcomes. The proposed method outperforms most of the state-of-the-art techniques and it is suitable to use in real-time ALPR applications.
\end{abstract}

Index Terms-ALPR, Bayesian theorem, Histogram, Localization, Negentropy, Probability Distributions

\section{INTRODUCTION}

The number of vehicles in transport systems has been constantly increasing and intelligent transportation system (ITS) assists the management and the control of these vehicles in efficient and swift ways. Automatic License Plate Recognition (ALPR) is one of the important technologies used by ITS to identify vehicles in cities and on roads. ALPR is divided mainly into two major parts: plate localization and recognition. ALPR consists of four main steps: data acquisition, plate localization, segmentation and Optical Character Recognition (OCR). The ALPR systems have to cope with many challenges such as different lightings and environmental conditions, and occluded plates. ITS is a real-time system and the ALPR process has to be sufficiently fast and with a high accuracy. However, with the presently implemented ALPR systems in complex backgrounds, the low-speed and the lack of high accuracy of these systems are much notable. As such, improving the performance and the accuracy rate of ALPR systems is still an essential research area in ITS. Therefore, developing a faster and more accurate method for License Plate Localization (LPL) becomes necessary.

In our previous publications [1], [2], we applied the saliency concept to ALPR for the first time and we presented a robust search-free LPL, incorporating hierarchical saliency. In this paper, based on some effective statistical methods, we present a novel algorithm to achieve a robust localization of multiple car number plates by measuring the distance between their pattern probability distributions. Section II describes some stateof-the-art methods of LPL. Then, in section III, the proposed method is discussed in details. Next, the experimental results and observations are evaluated in section IV. Finally, the paper is concluded in section V.

\section{RELATED WORK}

LPL aims to detect the License Plate (LP) within the scene. There are many different approaches on how to perform LPL. Some use the edge information [3], [4]. Others follow more complex algorithms such as neural networks [5], fuzzy logic [6], and other machine-learning methodologies [7]. Although current LPL algorithms have acceptable accuracies, they are time-consuming and their speed is not sufficiently high for real-time systems such as an ITS system. These algorithms use exhaustive search in the input image. The evaluation carried out in our research shows that the LP processing time of most of these methods is higher than $200-300 \mathrm{~ms}$, which is not acceptable for real-time ALPR applications. The most desirable amount of time taken to process each frame in an ITS systems is $50-80 \mathrm{~ms}$, such that no vehicles are missed in a high-speed highway. Current methods use search-based algorithms involving edge detection and Sliding Concentric Windows (SCW). A more robust approach requires the exploitation of various features from different modality data (image, video, edge map, and statistical features). It is believed that a new LPL method is required to meet the ITS necessities, that is being search-free, fast and robust to noise.

In our previous publication [1], a new saliency-based ALPR system was proposed. This search-free method accurately locates the LP based on the estimation of saliency, local variance and the use of Gabor filter. Also, recognition of patterns of numbers using $L_{1}-$ norm has been added to the traditional saliency detection method [2], [8].

A recent research carried out by Nejati et al. [9], proposed a new approach for localization of LP. In this method, similar to Safaei et al. [2] approach, down-sampling is the first step of the processing. Afterwards, noise removal and histogram equalization are performed. Then, to detect the candidate frame edges which are expected to contain the LP, the histogram of vertical edges followed by filtering is used to reduce the number of false detections. In the last step of the method by Nejati et al. [9] (the localization phase), the candidate plates are localized using the aspect ratio and the vertical projection of the histogram of edges. Although this approach has a reasonable accuracy of $95 \%$, its speed is not suitable for ITS applications. 
On the other hand, Abo et al. [10] introduced a new generic algorithm to detect the LP symbols. This method used an adaptive threshold approach that overcomes the dynamic changes of the light conditions in binary conversions. Connected Component Analysis (CCA) is then used to detect the candidate LP. Abo et al. have also introduced a novel scale-invariant geometric relationship matrix to adaptively detect different LP patterns. Moreover, Halin et al. [11] have proposed an LPL method that prepossesses the LPs from the front/rear view, followed by feature extraction from CCA and the use of a Naive Bayes classifier for the LPL. In a different approach, Salahshoor et al. [12] proposed an LPL method to overcome problems such as different weather conditions, skews, and occluded LPs. In this method, Bernsen thresholding is used as the main contribution. The LPL is done by horizontal projection and the use of Sobel filter followed by using fourdirectional distance-profile features to recognize the characters. Although this algorithm copes well with shadows and dirty LPs, its computation time and accuracy are not suitable for ITS systems. Anakavej et al. [13] have recently proposed a new method for LPL, based on Maximally-Stable Extremal Regions (MSERs). All candidate LP regions are classified in three categories of LP, LP background and other components by using aspect ratio, size and intensity. Finally, a rule-based decision is made to verify the candidate LPs. Anakavej et al. also use approaches such as symmetrical property, Pyramid Histogram-Oriented Gradients (PHOG), edge projection and visual saliency in LPL process. Although their algorithm has a high rate of accuracy, its speed rate requires to be further increased compared to the conventional methods such as [1] and [2], to suit an ITS application. In a recent study, Baharlou et al. [14] proposed an LPL method that is sensitive to angle, and a dynamic thresholding approach to overcome the probable drawbacks caused by different lighting conditions. Compared to the above methods, the accuracy and the speed of this method are appropriate though only the stationary backgrounds are handled by it.

Zeng et al. [15] recently proposed a new visual saliency detection model based on tensor sparse reconstruction. This method measures the saliency level of image regions using reconstructed residual, which achieves higher accuracy than the traditional sparse reconstruction based models. A stateof-art approach of saliency detection method, carried out by Li et al. [16] has also been proposed. This different saliency detection algorithm propagates coarse saliency based on robust background prior.

\section{PROPOSED METHOD}

In this research, we attempt to impose some conditions onto the saliency detection equations which are very diverse and include distances, occlusions, background, size, scale and direction changes. Given that the salient regions have known distributions, any new regions can be assessed based on its likelihood to such distributions. If the difference can be assumed to have Gaussian distribution, then, the conventional likelihood measure can be applied. For processing the images, there is no causality condition. So, we can accurately measure their probability distribution functions (PDFs). It is believed that, based on the distribution of the information, we can always examine whether there will be any significant changes in it. For the case of LPs, there are different distribution patterns inside and outside of LPs. In this paper, we have estimated the negentropy as the distance between distributions in the LPL process for the first time, resulting in high accuracy and high speed.

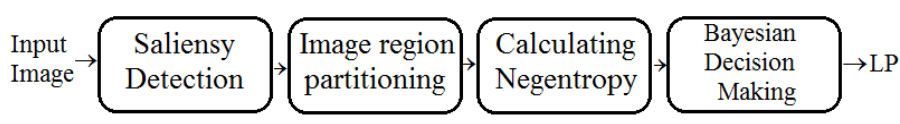

Fig. 1: The block diagram of the proposed method.

The block diagram of the proposed method is shown in Figure 1. As shown in this figure, in the first step, the salient region in the input image is divided into sub-images with the size of a $[9,17]$ window (experimentally selected for Persian LPs). Figure 2 shows the divided input image.

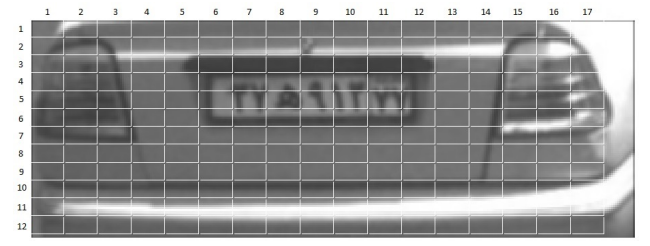

Fig. 2: The divided input image with window size of $[9,17]$.

Next, the histogram of each window is calculated. Figure 3 shows the histograms of four sample windows. Then, the entropy of each sub-image is calculated using Equation (1).
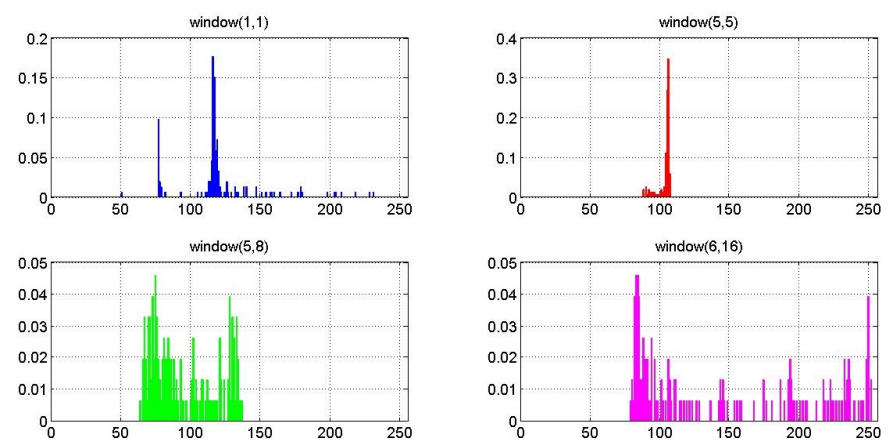

Fig. 3: Normalized histogram of sample windows.

Entropy of image sub-block $I$ can be defined as:

$$
H_{I}=-\sum_{i=0}^{255} p_{i} \log _{2}\left(p_{i}\right),
$$

where $p_{i}$ is the region PDF. The entropy values for Figure 2 is reported in Figure 4(a). Figure 4(b) shows these values in a 3-D graph. 
(a)
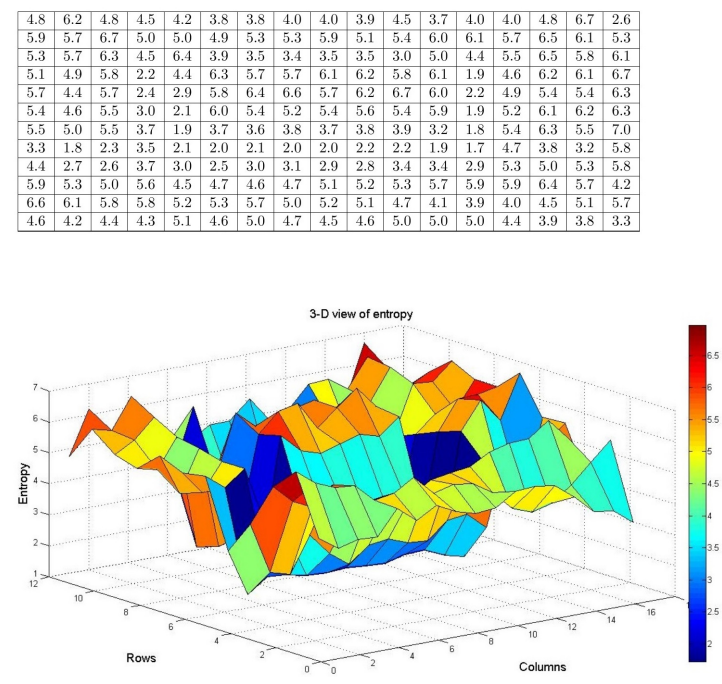

(b)

Fig. 4: (a) The entropy of each sub-image and (b) The 3-D view of the entropy values.

Algorithm 1 shows the pseudo-code of the proposed algorithm. In order to detect the LP candidates, we used Algorithm 2 to find the local maximum of the entropy matrix and, based on these local maxima, we determined the region of each candidate.

Figure 5 shows the detected candidates from the entropy matrix.
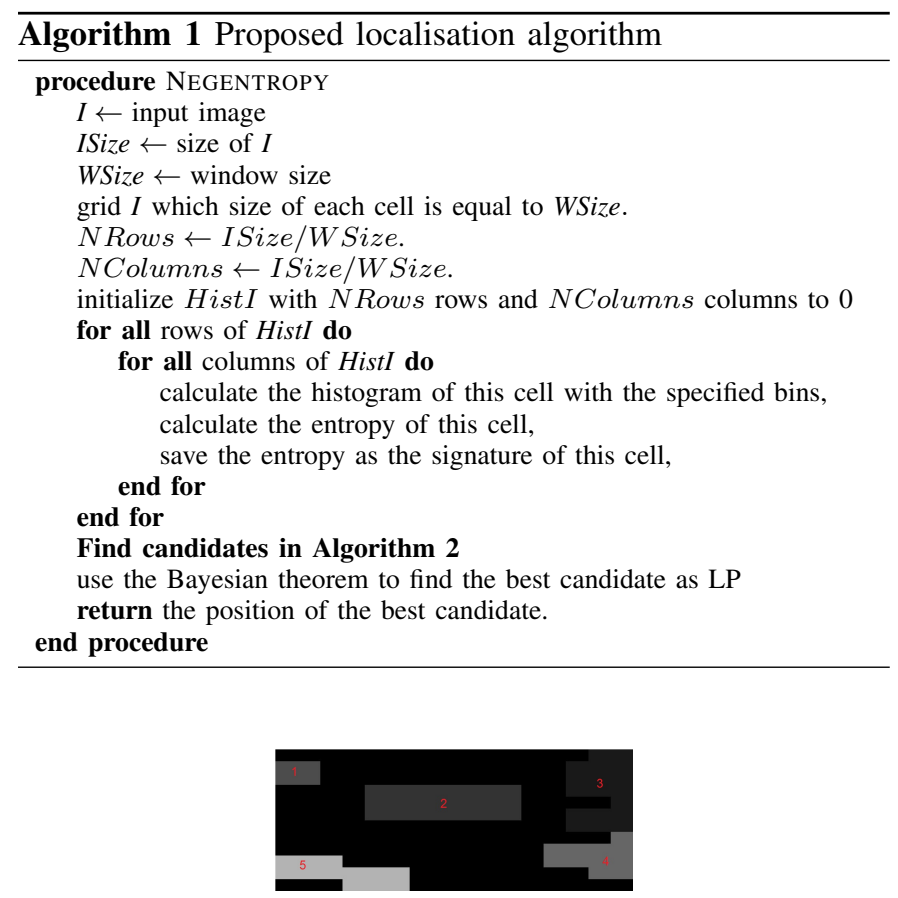

Fig. 5: The detected candidates of the entropy matrix using Algorthim 2.

Algorithm 2 shows the pseudo-code of the proposed algorithm for candidate detection based on local maximum. In this method the location of EntI is found. Then to evaluate whether the neighbourhood is part of the candidate region, four neighbourhoods of the regions with maximum pixel values are checked. Each pixel which is selected as part of the candidate, its neighbourhoods are checked as part of the candidate region.

When the candidate LPs are determined by Algorithm 2, the Bayesian probability method is used to calculate the probability for each candidate. The results of our proposed method is shown in Figure 6.

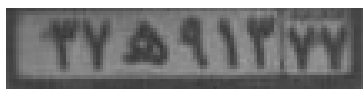

Fig. 6: The result of proposed method.

Based on our constraints and the Bayesian theorem, the LP will be selected from the detected candidates. The constraints (CON) are size (SIZ) and ratio (Rat). The Bayesian theorem is used to calculate the probability of each candidate (CAN) being an LP.

$P(\mathrm{CAN}$ is an $\mathrm{LP} \mid \mathrm{CON})=\frac{P(\mathrm{CON} \mid \mathrm{CAN} \text { is an } \mathrm{LP}) P(\mathrm{CAN} \text { is an } \mathrm{LP})}{P(\mathrm{CON})}$

There is no assumption about any candidate being an LP or not, so $P(\mathrm{CAN}$ is a $\mathrm{LP})=1 / 2$. Also, the denominator is a constant, so Equation (2) can be summarized as:

$$
P(\mathrm{CAN} \text { is an } \mathrm{LP} \mid \mathrm{CON}) \propto P(\mathrm{CON} \mid \mathrm{CAN} \text { is an } \mathrm{LP})
$$

The term $P(\mathrm{CON} \mid \mathrm{CAN}$ is an $\mathrm{LP})$ can be written as:

$$
\begin{aligned}
& P(\mathrm{CON} \mid \mathrm{CAN} \text { is an } \mathrm{LP})= \\
& P(\mathrm{SIZ} \mid \mathrm{CAN} \text { is an } \mathrm{LP}) \times P(\mathrm{RAT} \mid \mathrm{CAN} \text { is an } \mathrm{LP})
\end{aligned}
$$

where, $P(\mathrm{SIZ} \mid \mathrm{CAN}$ is an LP $)$ is considered to have uniform distributions between minimum and maximum sizes of the LP. We set these parameters to 1600 and 10000 pixels, respectively. Finally, we used Gaussian distribution for the ratio constraint.

$$
P(\mathrm{RAT} \mid \mathrm{CAN} \text { is an LP })=\mathcal{N}(2.5,0.25) .
$$

Table I shows the probabilities of each constraint for each object/region. By multiplying them together, the probability of each connected component can be calculated as shown in Table II. Based on the probabilities reported in Table II, the object with the label 2 in Figure 5 is selected as an LP.

\begin{tabular}{|c|c|c|}
\hline Object Number & $P($ SIZ $\mid$ CAN is a LP $)$ & $P($ RAT $\mid$ CAN is a LP $)$ \\
\hline 1 & 0 & $1.22 \mathrm{E}-8$ \\
\hline 2 & $2.27 \mathrm{E}-4$ & $4.56 \mathrm{E}-1$ \\
\hline 3 & 0 & $4.13 \mathrm{E}-11$ \\
\hline 4 & 0 & $9.59 \mathrm{E}-8$ \\
\hline 5 & 0 & $3.71 \mathrm{E}-7$ \\
\hline
\end{tabular}

TABLE I: The probability of each constraint for each object. 


\begin{tabular}{|c|c|c|}
\hline Object Number & $P(\mathrm{CON} \mid \mathrm{CAN}$ is a LP $)$ & Normalization \\
\hline 1 & 0 & 0 \\
\hline 2 & $1.04 \mathrm{E}-4$ & 1 \\
\hline 3 & 0 & 0 \\
\hline 4 & 0 & 0 \\
\hline 5 & 0 & 0 \\
\hline
\end{tabular}

TABLE II: The probability of all constraints for each object.

\section{EXPERIMENTAL RESULTS}

To evaluate our proposed algorithm, we tested the method on three datasets. First dataset has 43 images with an image size of $73 \times 275$ pixels. The images of this dataset were taken by an Infrared (IR) camera. The images have different viewpoints of LP and the position of the marginal pixel. The second dataset contains color images with 57 frames of size of $114 \times 384$ pixels. These images were taken by color camera in various viewpoints of LP and car colors. The third dataset was chosen from a public dataset [17], containing gray scale images with 340 frames of size of $340 \times 371$ pixels. Unlike the first and third datasets, where LPs are salient because of the IR reflection, in the second dataset, the images are converted to gray images and LPs are not sufficiently salient. Figure 7 shows sample images of the first, second and the third datasets.
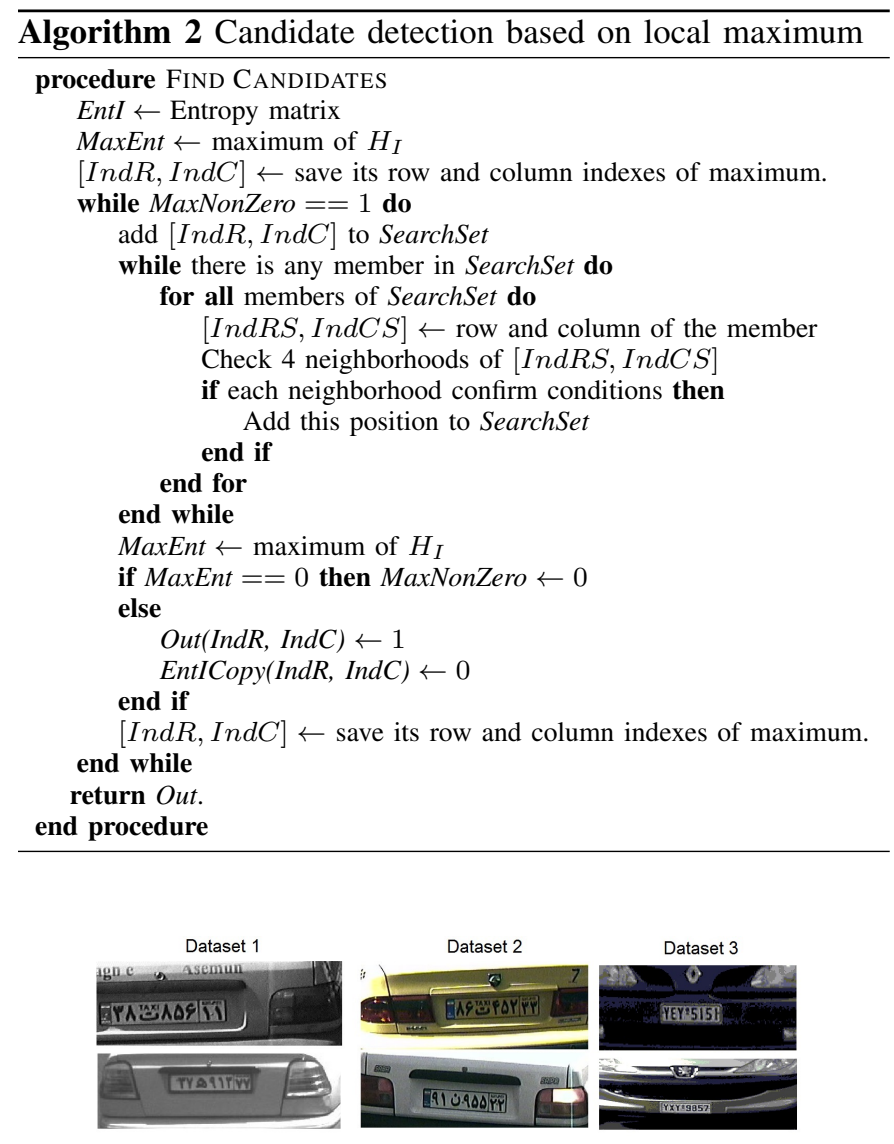

Fig. 7: Sample images of datasets.

Our proposed algorithm was tested on a Core-i7 CPU with the clock rates of $2.30 \mathrm{GHz}$. For evaluating this algorithm, we considered the Accuracy (ACCY) as the ratio of true results to the total data processed (when the coordinates of the LP are found), the Computation Time (CT) as the amount of time required to perform a computational process to get results, and the False Alarm Rate (FAR) as the ratio of false results to the total data processed (when a part of the image is reported to be an LP while it actually is not). Figure 8 shows the acceptable and unacceptable results.

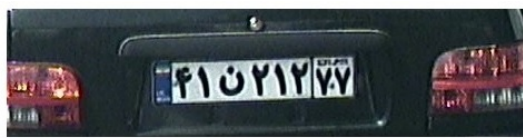

(a)

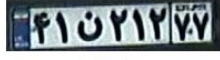

(b)

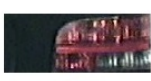

(c)
Fig. 8: Example of acceptable and unacceptable results. (a) the input image, (b) acceptable results, and (c) unacceptable results.

The results of applying our proposed method for these three datasets are shown in Figure 9. Table III shows the details of the experimental results for the proposed algorithm. As shown in this table, the ACCY of datasets 1 and 3 is higher than that of dataset 2. The FAR and the CT of datasets 1 and 3 are lower than those of dataset 2. The results show that, when the IR images in dataset 1 are tested with our proposed algorithm, their IR reflections increase the ACCY. This is mainly because of the conversion to gray-scale images, which is time-consuming.

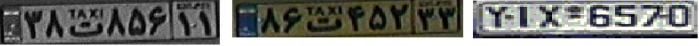

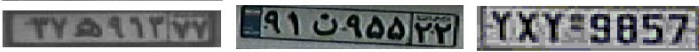

Fig. 9: Results of applying the proposed algorithm.

\begin{tabular}{|l|c|c|c|}
\hline Dataset & ACCY & FAR & CT (ms) \\
\hline 1 & $96 \%$ & $1 \%$ & 80 \\
\hline 2 & $90 \%$ & $4 \%$ & 174 \\
\hline 3 & $94.5 \%$ & $2.5 \%$ & 120 \\
\hline
\end{tabular}

TABLE III: Experimental results for the proposed algorithm using datasets 1,2 and 3 .

In order to evaluate the proposed algorithm, two other methods were also examined, both use the distance between the distributions, e.g. K-mean and energy metric. The experiments show that, although the methods which use K-mean do not need any thresholding, they are fast and robust, but require a learning algorithm and provides its best result when images in the dataset are distinct or well-separated from each other. Also, the K-mean clustering can divide an image into different clusters and it is a time-consuming algorithm. On the order hand, the energy metric calculates the distance between histograms. However, the ACCY of this method is low and its CT is high. Also, its detection contour may not be connected and the license plate may be divided into multiple objects. 
Figure 10 shows this comparison. As shown, the energy metric method has detected a wrong object as an LP and the K-mean has fairly detected the LP. However, our proposed method has correctly detected the right LP.

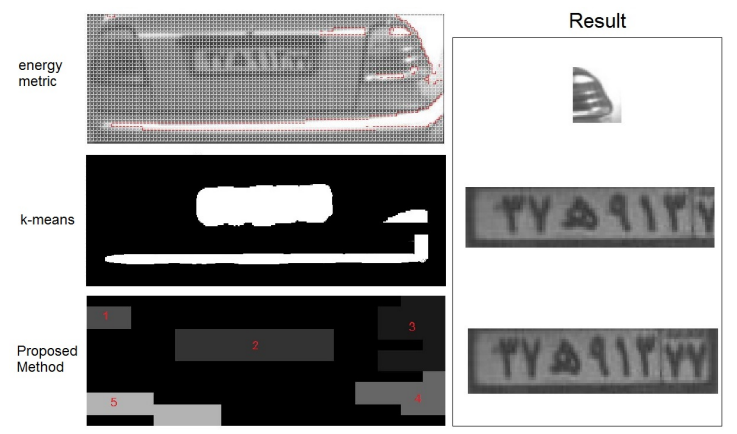

Fig. 10: Comparison of our proposed method to other methods for the calculation of distance between distributions.

Table IV shows the results of evaluating and comparing our proposed method to other recent methods using dataset 1 . As shown in this table, the superiority of our proposed methods in terms of accuracy and computation time is notable.

\begin{tabular}{c|c|c}
\hline Approach & Accuracy & Computation time (ms) \\
\hline \hline Safaei et al. 's [1] & $89 \%$ & 180 \\
\hline Safaei et al. 's [2] & $92 \%$ & 100 \\
\hline Nejati et al. 's [9] & $87 \%$ & 250 \\
\hline Anakavej et al. 's [13] & $71 \%$ & 320 \\
\hline K-means & $87 \%$ & 412 \\
\hline Energy metric & $80 \%$ & 423 \\
\hline Proposed algorithm & $96 \%$ & 80 \\
\hline \hline
\end{tabular}

TABLE IV: Comparison of our proposed metohd to other current methods using dataset 1 .

\section{CONCLUSIONS}

In this paper, we incorporated negentropy as a suitable statistical distance measure, into the saliency-based searchfree LPL. This approach uses the histogram (PDF) of subimages, calculates the entropy of each sub-image and finds the candidate LPs. Then, using the Bayesian theorem, the LPL process is performed. The proposed method shows a high accuracy $(96 \%)$ and low computation time $(80 \mathrm{~ms})$ per plate, which is a significant improvement over traditional benchmark techniques. For the future work, we will investigate the effects of LP rotation angle on the localization accuracy, the use of adaptive window sizes for image partitioning and using more complicated datasets to overcome the problem of tuning the system parameters.

\section{REFERENCES}

[1] A. Safaei, H. Tang, and S. Sanei, "Search-free license plate localization based on saliency and local variance estimation," in Seventh International Conference on Machine Vision (ICMV 2014), pp. 94451U_ 94451U, International Society for Optics and Photonics, 2015.

[2] A. Safaei, H. Tang, and S. Sanei, "Robust search-free car number plate localization incorporating hierarchical saliency," Journal of Computer Science and Systems Biology, vol. 9, pp. 093-103, June 2016.
[3] G. Garibotto, P. Castello, and E. D. Ninno, "Dynamic vision for license plate recognition," in Multimedia Video-Based Surveillance Systems, vol. 573 of The Springer International Series in Engineering and Computer Science, pp. 272-283, Springer US, 2000.

[4] C. N. E. Anagnostopoulos, L. E. Anagnostopoulos, V. Loumos, and E. Kayafas, "A license plate-recognition algorithm for intelligent transportation system applications," Trans. Intell. Transport. Sys., vol. 7, pp. 377-392, Sept. 2006.

[5] C.-M. Wang and J.-H. Liu, "License plate recognition system," in Fuzzy Systems and Knowledge Discovery (FSKD), 2015 12th International Conference on, pp. 1708-1710, Aug 2015.

[6] D. Kassymkhanova, D. Kurochkin, N. Denissova, S. Kumargazhanova, and A. Tlebaldinova, "Majority voting approach and fuzzy logic rules in license plate recognition process," in Application of Information and Communication Technologies (AICT), 2014 IEEE 8th International Conference on, pp. 1-5, Oct 2014.

[7] S. Subhadhira, U. Juithonglang, P. Sakulkoo, and P. Horata, "License plate recognition application using extreme learning machines," in Student Project Conference (ICT-ISPC), 2014 Third ICT International, pp. 103-106, March 2014.

[8] L. Itti, C. Koch, and E. Niebur, "A model of saliency-based visual attention for rapid scene analysis," IEEE Trans. Pattern Anal. Mach. Intell., vol. 20, pp. 1254-1259, Nov. 1998.

[9] M. Nejati, A. Majidi, and M. Jalalat, "License plate recognition based on edge histogram analysis and classifier ensemble," in 2015 Signal Processing and Intelligent Systems Conference (SPIS), pp. 48-52, Dec 2015.

[10] G. A. Samra and F. Khalefah, "Localization of license plate number using dynamic image processing techniques and genetic algorithms," IEEE Transactions on Evolutionary Computation, vol. 18, pp. 244-257, April 2014.

[11] A. A. Halin, N. M. Sharef, A. H. Jantan, and L. N. Abdullah, "License plate localization using a naive bayes classifier," in Signal and Image Processing Applications (ICSIPA), 2013 IEEE International Conference on, pp. 20-24, Oct 2013.

[12] M. Salahshoor, A. Broumandnia, and M. Rastgarpour, "Application of intelligent systems for iranian license plate recognition," in Intelligent Systems (ICIS), 2014 Iranian Conference on, pp. 1-6, Feb 2014.

[13] T. Anakavej, A. Kawewong, and K. Patanukhom, "License plate localization using msers and vehicle frontal mask localization using visual saliency for vehicle recognition," in Digital Information and Communication Technology and it's Applications (DICTAP), 2014 Fourth International Conference on, pp. 31-36, May 2014.

[14] S. M. Baharlou, S. Hemayat, A. Saberkari, and S. Yaghoobi, "Fast and adaptive license plate recognition algorithm for persian plates," in Pattern Recognition and Image Analysis (IPRIA), 2015 2nd International Conference on, pp. 1-6, March 2015.

[15] Y. Zeng and Y. Xu, "Saliency detection using tensor sparse reconstruction residual analysis," in 2016 IEEE International Conference on Acoustics, Speech and Signal Processing (ICASSP), pp. 2961-2965, March 2016.

[16] C. Li, P. Yang, and H. Sheng, "Robust saliency propagation based on random walks," in 2016 IEEE International Conference on Acoustics, Speech and Signal Processing (ICASSP), pp. 1821-1825, March 2016.

[17] "Medialab LPR database." http://www.medialab.ntua.gr/research/LPRdatabase.html, 2009. 